\title{
Exploring the Bone Proteome to Help Explain Altered Bone Remodeling and Preservation of Bone Architecture and Strength in Hibernating Marmots
}

\author{
Alison H. Doherty ${ }^{1}$ \\ Danielle M. Roteliuk ${ }^{2}$ \\ Sara E. Gookin ${ }^{1}$ \\ Ashley K. McGrew ${ }^{3}$ \\ Carolyn J. Broccardo ${ }^{4}$ \\ Keith W. Condon ${ }^{5}$ \\ Jessica E. Prenni ${ }^{4}$ \\ Samantha J. Wojda ${ }^{1}$ \\ Gregory L. Florant ${ }^{2}$ \\ Seth W. Donahue ${ }^{1, \star}$ \\ ${ }^{1}$ Department of Mechanical Engineering, Colorado State \\ University, Fort Collins, Colorado; ${ }^{2}$ Department of Biology, \\ Colorado State University, Fort Collins, Colorado; ${ }^{3}$ Veterinary \\ Diagnostics Laboratory, Colorado State University, Fort \\ Collins, Colorado; ${ }^{4}$ Proteomics and Metabolomics Facility \\ and Department of Biochemistry and Molecular Biology, \\ Colorado State University, Fort Collins, Colorado; \\ ${ }^{5}$ Department of Anatomy and Cell Biology, Indiana \\ University School of Medicine, Indianapolis, Indiana
}

Accepted 5/10/2016; Electronically Published 6/29/2016

\begin{abstract}
Periods of physical inactivity increase bone resorption and cause bone loss and increased fracture risk. However, hibernating bears, marmots, and woodchucks maintain bone structure and strength, despite being physically inactive for prolonged periods annually. We tested the hypothesis that bone turnover rates would decrease and bone structural and mechanical properties would be preserved in hibernating marmots (Marmota flaviventris). Femurs and tibias were collected from marmots during hibernation and in the summer following hibernation. Bone remodeling was significantly altered in cortical and trabecular bone during hibernation with suppressed formation and no change in resorption, unlike the increased bone resorption that occurs during disuse in humans and other animals. Trabecular bone architecture and cortical bone geometrical and mechanical properties were not different between hibernating and active marmots, but bone marrow adiposity was significantly greater in hibernators. Of the 506 pro-
\end{abstract}

*Corresponding author; e-mail: seth.donahue@colostate.edu.

Physiological and Biochemical Zoology 89(5):364-376. 2016. (C) 2016 by The University of Chicago. All rights reserved. 1522-2152/2016/8905-5168\$15.00. DOI: $10.1086 / 687413$ teins identified in marmot bone, 40 were significantly different in abundance between active and hibernating marmots. Monoaglycerol lipase, which plays an important role in fatty acid metabolism and the endocannabinoid system, was 98-fold higher in hibernating marmots compared with summer marmots and may play a role in regulating the changes in bone and fat metabolism that occur during hibernation.

Keywords: hibernation, yellow-bellied marmots, bone remodeling, bone proteomics.

\section{Introduction}

Periods of physical inactivity typically accelerate bone resorption and unbalance bone resorption from formation, leading to bone loss, decreased mechanical properties, and increased fracture risk (McGee-Lawrence et al. 2008). As little as 2 wk of mechanical unloading of the skeleton has been shown to result in bone loss in rats ( $\mathrm{Li}$ et al. 1990). Extensive periods of disuse in dogs lead to substantial deficits in bone properties, and it takes a twofold or longer recovery period to restore bone properties to baseline levels (Kaneps et al. 1997). Bone loss due to mechanical unloading has also been shown to occur in mice, turkeys, monkeys, and sheep (Young et al. 1983; Rubin et al. 1988, 1996; Rantakokko et al. 1999). Animals that hibernate (e.g., marmots and bears) are naturally physically inactive for extended periods ( 6 mo or longer) annually. However, bears, marmots, woodchucks, and ground squirrels maintain bone properties, despite these long bouts of physical inactivity (McGee et al. 2007b, 2008; McGee-Lawrence et al. 2009a, 2011; Utz et al. 2009; Doherty et al. 2012; Wojda et al. 2012). Hibernating mammals demonstrate remarkable resilience by having evolved physiological mechanisms that allow them to survive extreme physiological and environmental conditions for prolonged periods of time (Carey et al. 2003). Physiological processes in bone have evolved to produce many unique adaptations in response to different mechanical environments, including the preservation of bone mechanical properties during prolonged mechanical unloading in mammalian hibernators (Doherty et al. 2015). The preservation of bone mechanical properties promotes survival of mammalian hibernating species by allowing them to resume feeding and reproductive activities following hibernation without risk of bone fracture.

To conserve metabolic energy during hibernation, winter hibernating mammals reduce basal metabolism to between $2 \%$ and $5 \%$ 
(small rodent hibernators) and to 25\% (bears) of the basal metabolic rates they experience during periods of physical activity when not hibernating in the summer (Carey et al. 2003; Toien et al. 2011). In bears, the reduction in overall metabolism is similar to the reduced rate of bone metabolism that occurs during hibernation. In grizzly bears (Ursus arctos horribilis), the activation of new intracortical remodeling sites during hibernation was reduced to $25 \%$ of summer levels (McGee et al. 2008), similar to metabolic rate reducing to $25 \%$ of summer levels in hibernating bears (Toien et al. 2011). Suppressed bone remodeling in bears is also supported by studies using serum markers of bone turnover (McGee-Lawrence et al. 2015). Since bears do not ingest or excrete calcium during hibernation (Nelson et al. 1984), normal serum calcium concentrations during hibernation (Floyd et al. 1990; Seger et al. 2011) suggest that bone resorption and formation are balanced during hibernation, as histological indices also suggest (McGee et al. 2008; McGee-Lawrence et al. 2009b). Thus, reduced and balanced bone turnover contributes to the prevention of bone loss and the preservation of bone strength during hibernation. This is in contrast to what happens in other animals, where disuse leads to increased bone turnover, bone loss, and reduced mechanical properties (Li et al. 2005; Baek and Bloomfield 2009). How the rate of bone remodeling changes in hibernating rodents - such as marmots, in which basal metabolic rates drop to $2 \%-5 \%$ of summer levels during hibernation-is unknown. Since bone remodeling is a metabolically expensive process (Ishii et al. 2009), we hypothesized bone remodeling in marmots would be reduced during hibernation similar to reductions in overall metabolic rate.

The purpose of this study was to quantify differences in bone remodeling, architecture, mechanical properties, and protein expression profiles in hibernating and active summer yellow-bellied marmots (Marmota flaviventris). Like bears, marmots do not lose bone mass and strength during hibernation periods lasting 56 mo (Wojda et al. 2012). Marmots decrease their basal metabolic rate during hibernation to $5.5 \%$ of the levels observed during periods of physical activity (Hock 1969). We hypothesized that, like bears, marmots reduce the rate of bone remodeling during hibernation by percentages that are similar to reductions in their metabolic rate and that they maintain balanced bone formation and resorption. These changes are expected to preserve bone architectural and mechanical properties during hibernation. Additionally, to increase our understanding of potential mechanisms involved in regulating bone metabolism during hibernation, we quantified changes in bone marrow adipocytes and bone protein levels in summer and winter hibernating marmots.

\section{Material and Methods}

Animals

All procedures were conducted with prior approval from the Colorado State University Institutional Animal Care and Use Committee (protocol 12-3313A). Marmot trapping permits were acquired from the Colorado Department of Natural Resources (permit 13TR099). Healthy marmots (Marmota flaviventris) were trapped with Have-a-Heart live traps from the Front Range and Rocky Mountain areas surrounding Fort Collins, Colorado, in the spring/summer of 2012. Age was determined by body weight, as previously described (Armitage et al. 2003). A total of 10 marmots were collected (table 1) and transported to Colorado State University. The marmots were identified by sex and age (two age groups: $<1 \mathrm{yr}$ and $1 \mathrm{yr}$ or older) at the time of capture. They were further divided into two experimental groups: (1) January hibernating marmots and (2) June active marmots (table 1). The June active marmots were allowed to hibernate through March 25 and then be physically active for nearly 3 mo before sampling in June.

Marmots were housed individually in an approved environmental chamber continuously adjusted to the local Colorado photoperiod. Animals were fed rodent chow (Teklad Global 18\% Protein Rodent Diet) and water ad lib. The temperature of the environmental chamber was dropped to $20^{\circ} \mathrm{C}$ in preparation for the hibernation season on August 1, 2012, and subsequently lowered gradually to reach a temperature of $4^{\circ} \mathrm{C}\left( \pm 1^{\circ}-\right.$ $2^{\circ} \mathrm{C}$ ). Marmots were maintained at $4^{\circ} \mathrm{C}$ and kept in constant darkness for the duration of the hibernation season (SeptemberMarch). Animals started entering torpor in November and were allowed to hibernate through January. Torpor bouts were synchronized on January 10, 2013, to ensure that each marmot was in torpor during calcein injections and sample collection time points. Synchronization was accomplished by bringing the marmots to room temperature until nonshivering thermogenesis was initiated within 1-2 h. Animals were then checked within $3 \mathrm{~h}$ to determine whether they could lift their head and were alert before allowing animals to resume torpor, now synchronized as a group. Five marmots were removed from the room at the end of January for sampling (January hibernating marmots) and the other five (June active marmots) were allowed to continue hibernating. At the end of the hibernation season in March, the temperature of the room was elevated to $10^{\circ} \mathrm{C}$ and gradually increased to $20^{\circ} \mathrm{C}$ to arouse the remaining five marmots from hibernation and to encourage normal physical activity and feeding until euthanasia in June.

\section{Calcein Labeling}

Calcein, a bone-labeling fluorochrome, was administered to hibernating (January) and active (June) marmots to determine the rate of bone mineralization between seasons. In January, five hibernating marmots were subcutaneously injected with calcein $(10 \mathrm{mg} / \mathrm{kg}$ at $10 \mathrm{mg} / \mathrm{mL})$ between the shoulder girdles, using a 25-23-gauge needle, $13 \mathrm{~d}$ before euthanasia. This pro-

Table 1: Age and sex of marmots for each season

\begin{tabular}{lcccccc}
\hline & \multicolumn{2}{c}{ Males } & & \multicolumn{2}{c}{ Females } \\
\cline { 2 - 3 } \cline { 6 - 7 } \cline { 5 - 7 } & Subadult & Adult & & Subadult & Adult \\
\hline January hibernating & 1 & 0 & & 1 & 3 \\
June active & 0 & 1 & & 1 & 3 \\
\hline
\end{tabular}

Note. Subadults were $<1 \mathrm{yr}$ old. Adults were $>1$ yr old. 
cedure was repeated, alternating sides of the shoulder girdle, $3 \mathrm{~d}$ before euthanasia for the second label. These five marmots were euthanized on January 28. All five marmots were in torpor at the time of each calcein injections and at the time of euthanasia. Three months after emergence from hibernation in June, the remaining five (physically active) marmots were euthanized. The same calcein labeling regimen was performed in June on the fully active marmots that was conducted in January. Following euthanasia, the femurs and tibias were immediately dissected and stored for investigation of differences in bone metabolism and properties between seasons by microcomputed tomography $(\mu \mathrm{CT})$, histomorphometry, proteomics, and material testing.

\section{Microcomputed Tomography}

The left femurs were collected and fixed in $10 \%$ neutral buffered formalin for $48 \mathrm{~h}$ and stored in $70 \%$ ethanol. After fixation, these femurs were scanned using a $\mu \mathrm{CT}$ scanner (SCANCO $\mu \mathrm{CT} 80$ Medical, Switzerland). The bones were held vertically with small foam cubes in a $35-\mathrm{mm}$ holder filled with $70 \%$ ethanol. Scans were acquired using $70 \mathrm{kVp}, 114 \mu \mathrm{A}$, and $8 \mathrm{~W}$ at a medium resolution and slice thickness of $36 \mu \mathrm{m}$. The start of the scan region was defined as $0.32 \mathrm{~mm}(\sim 4.3 \%$ of average femoral length) proximal to the distal physis (fig. $1 A$ ). The scan region spanned $2.0 \mathrm{~mm}$ of the distal metaphysis. Trabecular bone regions of interest were drawn approximately two to three trabecular thicknesses within the cortical shell for analysis of trabecular properties, using the SCANCO software (fig. 1B). Measurements of interest included trabecular bone volume fraction (\%), trabecular tissue mineral density (mg hydroxyapatite $\left./ \mathrm{cm}^{3}\right)$, trabecular thickness $(\mathrm{mm})$, trabecular number $(1 / \mathrm{mm})$, and trabecular separation $(\mathrm{mm})$.

\section{Trabecular Histomorphometry}

The distal femoral metaphyses were serial sectioned longitudinally in resin blocks at a thickness of $4 \mu \mathrm{m}$ and mounted on glass slides with Eukitt mounting media (Electron Microscopy Sciences, Hatfield, PA). Sections were stained with von Kossa/ MacNeal's tetrachrome or tartrate-resistant acid phosphatase (TRAP) or left unstained to examine calcein fluorochrome labeling. The region of evaluation matched the $\mu \mathrm{CT}$ region of interest; that is, $2 \mathrm{~mm}$ of the metaphysis were analyzed beginning $0.32 \mathrm{~mm}$ proximal to the distal physis. The von Kossa/ MacNeal's stained slides (imaged at $\times 400$ magnification) provided information regarding osteoblast activity, as measured by osteoid width, surface, and osteoblast number along the trabecular bone surface (BS; fig. 2A). The percentage of marrow area occupied by the area of adipocytes was also quantified in the Kossa/MacNeal's slides. Osteoclast measures were made at $\times 200$ magnification using TRAP-stained slides (fig. $2 B$ ) to quantify the osteoclast number, osteoclast surface, and eroded surface normalized by trabecular BS. Slides reserved for fluorescence imaging were examined at $\times 100$ magnification to quantify mineralization parameters (Dempster et al. 2013), length of single (calcein)labeled surface (sLS/BS), length of double-labeled surface (dLS/ $\mathrm{BS})$, mineralizing surface $(\mathrm{MS} / \mathrm{BS}=(\mathrm{dLS}+\mathrm{sLS} / 2) / \mathrm{BS})$, and mineral apposition rate (MAR). Digital images were captured with an Olympus BX61VS slide scanner and associated Hamamatsu Orca- $\mathrm{R}^{2}$ digital camera. Sections were scanned in EFI mode and converted to tiffs using the Olyvia VS-Workspace software.


Figure 1. A, Microcomputed tomography scout view of the marmot distal femoral metaphysis. Scans were initiated $0.32 \mathrm{~mm}$ proximal to the distal physis. The scan region included $2.0 \mathrm{~mm}$ of the distal metaphysis. $B$, The region of interest was drawn two to three trabecular thicknesses inside the endosteal aspect of the distal metaphysis for analysis (outlined region). A color version of this figure is available online. 


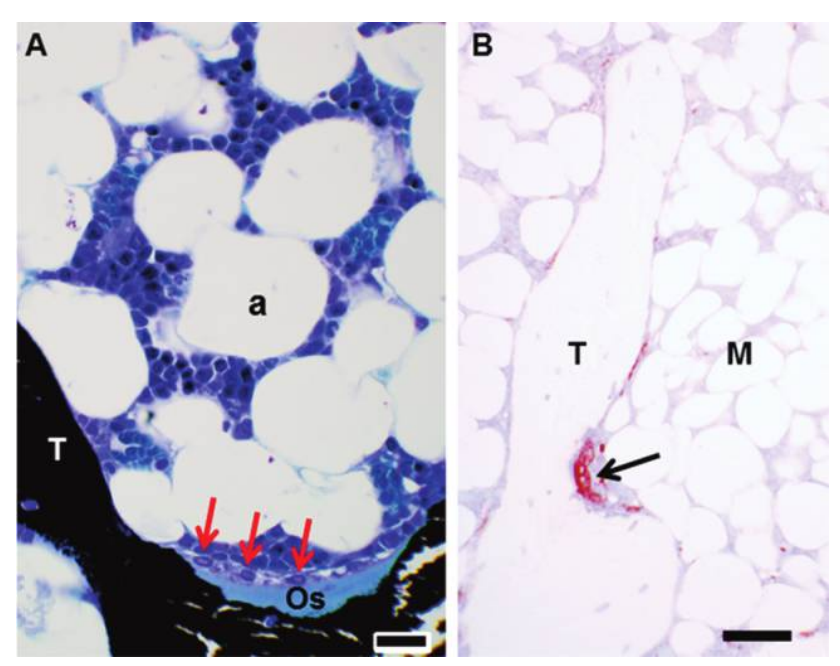

Figure 2. A, Distal femoral metaphysis stained with von Kossa/ MacNeal's tetrachrome. Black indicates mineralized trabecular bone (T), light blue represents osteoid (OS), and osteoblasts can be seen on the surface of the osteoid (arrows). Bone marrow filled with adipocytes (a) surrounds the trabeculae. Scale bar $=20 \mu \mathrm{m}, \times 400 \mathrm{mag}$ nification. $B$, Tartrate-resistant acid phosphatase (red) fills a multinucleated osteoclast (arrow) over the eroded surface of a trabecular strut $(\mathrm{T})$ surrounded by marrow $(\mathrm{M})$. Scale bar $=50 \mu \mathrm{m}, \times 200$ magnification.

All slides (cortical and trabecular sections) were analyzed using Bioquant software (ver 12.1.6; Bioquant Image Analysis, Nashville). One slide from each animal for each stain was analyzed.

\section{Cortical Histomorphometry}

Following $\mu \mathrm{CT}$ scanning, the left femurs were cut into segments to isolate the diaphysis and distal metaphysis. These segments were individually embedded in methylmethacrylate-containing resin blocks. The midshafts were sectioned $(\sim 100 \mu \mathrm{m})$ in cross section using a Buehler Isomet 1000 precision saw (112180; Buehler, Lake Bluff, IL), and the sections were mounted onto slides using Eukitt mounting media. Sections were ground to a thickness of $\sim 80 \mu \mathrm{m}$ using a Buehler Metaserv 250 GrinderPolisher (4910055; Buehler) using 600-800-grit pressure-sensitive adhesive abrasive paper (Buehler). Whole cortical cross sections were imaged using a Nikon Eclipse i80 microscope to investigate osteocyte lacunar area. The cortices were divided into four anatomical quadrants: anterior, medial, posterior, and lateral (fig. $3 A$ ). One image per quadrant was acquired at $\times 400$ magnification with an Olympus DP71 digital camera and the associated Olympus software (cellSens Entry). Total lacunar number, individual lacunar area, lacunar porosity, and lacunar density were measured using Bioquant Osteo software (ver. 12.1.6; Bioquant Image Analysis) and compared between seasons.

The cortices were also inspected under fluorescent light using an FITC filter with the same microscope. Images were acquired at $\times 100$ magnification to investigate the periosteal and endosteal surfaces of the cortical midshafts and to determine periosteal and endosteal MARs (fig. 3B). Other measurements of interest were periosteal and endosteal sLS and dLS relative to total BS.

\section{Three-Point Bending}

The right femur was collected from each animal at the time of euthanasia, cleaned of soft tissue, wrapped in wet paper towels, and stored at $-80^{\circ} \mathrm{C}$. The day of mechanical testing, the right femurs were thawed and rehydrated in $0.9 \%$ saline for $5 \mathrm{~h}$ before bending tests. The bones were broken in three-point bending to quantify the mechanical properties of the femurs in hibernating and summer active marmots. Femurs were loaded to failure using a MTS Bionix Tabletop tester (model 370.02; Eden Prairie, MN) with a 2,000-lb load cell (Interface, 1210AF-2k). Two rounded fixtures (7.4-mm diameter) were used to support the distal metaphysis and femoral neck, with an average span of $37 \mathrm{~mm}$ between the supports. The bones were held in place by a small preload of 5-10 $\mathrm{N}$ to avoid slippage while applying load via the rounded loader attached to the crosshead actuator. The femurs were loaded with the anterior surface of the diaphyseal midshaft in tension at a rate of $10 \mathrm{~mm} / \mathrm{min}$ (McGee-Lawrence et al. 2011). Cross-sectional properties were obtained from histological thick sections at $\times 10$ magnification of the left femoral midshaft using a custom macro in Scion Image (ver. 4.0.3.2). Three-point bending data and cross-sectional properties were analyzed using a custom MATLAB code (MathWorks, ver. R2013b) by asymmetric beam theory, as previously described (McGeeLawrence et al. 2009a).

\section{Proteomics}

Right tibias were flash frozen immediately at harvest from the euthanized marmots. To extract proteins from marmot bones, tibias were thawed, and all remaining soft tissue was removed. A 3-mm section of the proximal metaphysis was cut from the bone, and the cortical shell was removed using a dremel, leaving only the trabecular bone. The trabecular bone was then cut into three sections, each approximately $1 \mathrm{~mm}$ thick, and sonicated to remove marrow, blood, and other tissues. Proteins were extracted according to a previously published protocol (Jiang et al. 2007). Briefly, the cleaned bones were placed into phosphate buffered saline containing $1 \%$ protease inhibitor cocktail (Thermo Scientific, PI 78415) overnight at room temperature. The bones were transferred to new vials containing $500 \mu \mathrm{L}$ of $1.2 \mathrm{M}$ hydrochloric acid (Fisher, A144) and incubated overnight at $4^{\circ} \mathrm{C}$. The supernatant was collected as the first protein extraction. The bones were rinsed with Milli-Q water and covered with $500 \mu \mathrm{L}$ of $100 \mathrm{mM}$ Tris (Fisher, T393) and $6 \mathrm{M}$ guanidine- $\mathrm{HCl}$ (Fisher, BP178) for $72 \mathrm{~h}$ at $4^{\circ} \mathrm{C}$. The supernatant was then collected as extract 2 , the bones were rinsed, and $100 \mathrm{mM}$ Tris, $6 \mathrm{M}$ guanidine$\mathrm{HCl}$, and $0.5 \mathrm{M}$ tetrasodium ethylenediaminetetraacetic acid (Sigma, ED4S) were added to the bone vials for $72 \mathrm{~h}$, with an incubation temperature of $4^{\circ} \mathrm{C}$. This solution was collected as extract 3 , and the bones were rinsed and soaked in $6 \mathrm{M} \mathrm{HCl}$ 

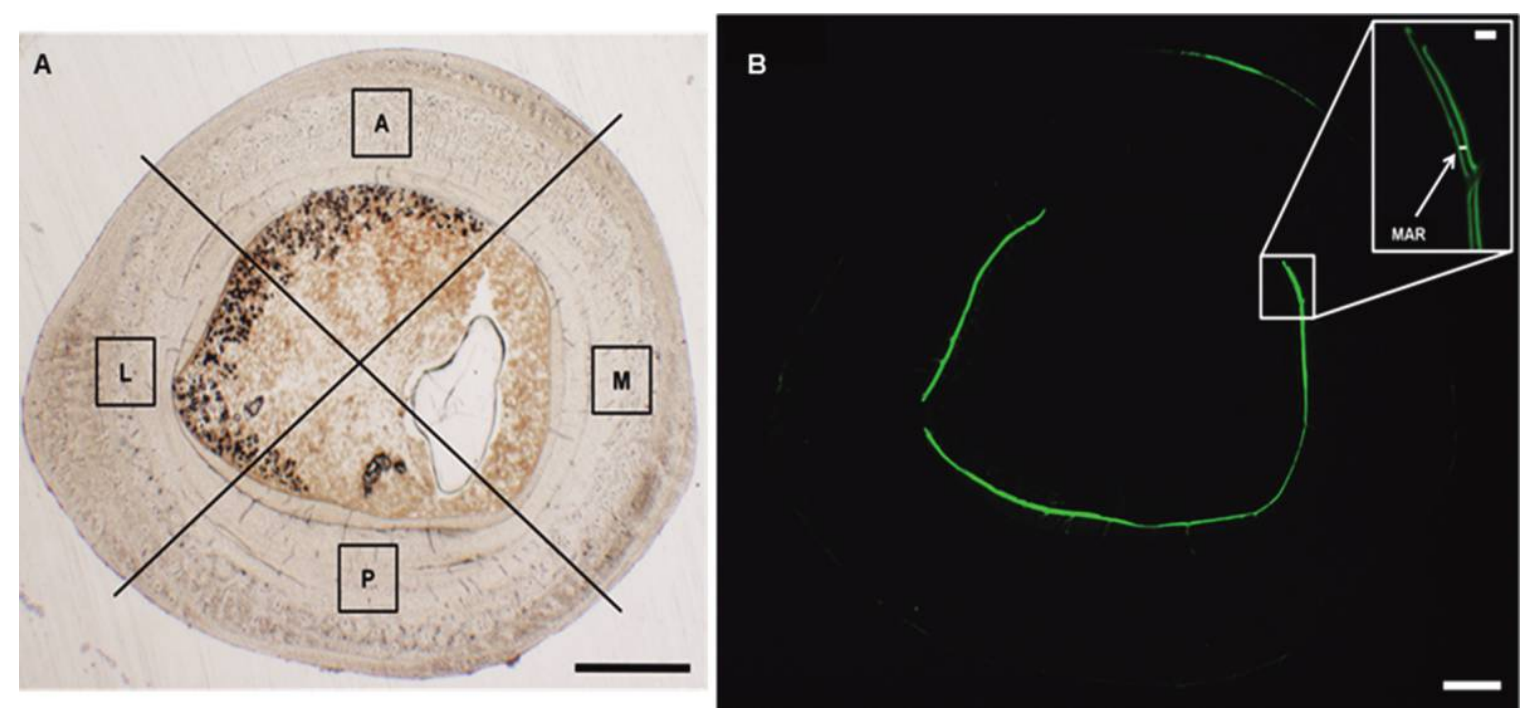

Figure 3. Cortical cross section of the marmot femoral midshaft. $A$, Cortices were divided into quadrants (A, anterior; $\mathrm{M}$, medial; $\mathrm{P}$, posterior; $\mathrm{L}$, lateral) and imaged near the center of the cortex (bounding box) at $\times 400$ magnification to determine osteocyte lacunar area. Scale bar $=$ $1 \mathrm{~mm}$. B. Same midshaft under fluorescent light at $\times 20$ magnification. Scale bar $=0.5 \mu \mathrm{m}$. Inset shows the interlabel width (horizontal line) between two endosteal calcein labels that were given $10 \mathrm{~d}$ apart. Mineral apposition rate (MAR) is calculated as interlabel width divided by time between labels $($ MAR $=$ interlabel width/10 d). Scale bar $=50 \mu \mathrm{m}$.

overnight at $4^{\circ} \mathrm{C}$ in order to collect the final protein extract solution, extract 4 .

Protein concentration of each extract (1-4) was determined using a standard Peirce BCA kit (Thermo Scientific, 23227). Thirty milligrams of each protein extract were precipitated by two acetone precipitation steps at $-20^{\circ} \mathrm{C}$. Proteins were denatured using urea (Sigma-Aldrich, U5378), reduced with dithiothreitol (Bio-Rad, 161-0611), and alkylated with iodoacetamide (Sigma-Aldrich, I6125). Protein digestion was carried out with ProteaseMAX Surfactant (Promega, V2071) and Trypsin Gold (mass spectrometry grade, Promega, V5280). The extracts were purified and concentrated using Pierce C18 spin columns (Thermo Scientific, 89873), dried, and resuspended in 3\% acetonitrile/ $0.1 \%$ formic acid. Tryptic peptides were purified and concentrated using an online enrichment column (Thermo Scientific, $5 \mu \mathrm{m}, 100-\mu \mathrm{m}$ inner diameter [ID] $\times 2 \mathrm{~cm} \mathrm{C18} \mathrm{column).}$ Subsequent chromatographic separation was performed on a reverse phase nanospray column (Thermo Scientific EASYnano-LC, $3 \mu \mathrm{m}, 75-\mu \mathrm{m}$ ID $\times 100 \mathrm{~mm}$ C18 column) using a 90-min linear gradient from $10 \%-30 \%$ buffer B (100\% acetonitrile, $0.1 \%$ formic acid) at a flow rate of $400 \mathrm{~nL} / \mathrm{min}$. Peptides were eluted directly into the mass spectrometer (Thermo Scientific Orbitrap Velos), and spectra were collected over a mass/charge range of 4002,000 Da, using a dynamic exclusion limit of $2 \mathrm{MS} / \mathrm{MS}$ spectra of a given peptide mass (exclusion duration of $90 \mathrm{~s}$ ). The instrument was operated in Orbitrap linear trap quadropole (LTQ) mode, where precursor measurements were acquired in the orbitrap (60,000 resolution), and MS/MS spectra (top 20) were acquired in the LTQ ion trap. Compound lists of the resulting spectra were generated using Xcalibur 2.2 software (Thermo Scientific) with an $\mathrm{S} / \mathrm{N}$ threshold of 1.5 and 1 scan/group. For quality control, instrument functionality and stability was monitored using the Mass QC software (Proteome Software; Rudnick et al. 2010). Quality control samples were injected at least once every $24 \mathrm{~h}$ during the analysis, and the data were analyzed using the Mass QC software. Values for all metrics were within normal limits throughout the duration of the experiment, indicating instrument stability and data robustness.

MS/MS spectra were searched against the ground squirrel protein database (as constructed by Shao et al. [2010]) concatenated to a reverse database (38,594 sequence entries), using the Mascot database search engine (ver. 2.3). A $0.07 \%$ peptide false discovery rate was calculated by Scaffold on the basis of hits to the reverse database (Kall et al. 2008). Search parameters were as follows: monoisotopic mass, parent ion mass tolerance of $20 \mathrm{ppm}$, fragment ion mass tolerance of $0.8 \mathrm{Da}$, fully tryptic peptides with three missed cleavages, variable modification of oxidation of $\mathrm{M}$, carbamylation of CKMR, and carbamidomethylation of C. Search results were imported and combined using probabilistic protein identification algorithms (Keller et al. 2002) implemented in Scaffold software (ver. 4, Proteome Software, Portland, OR; Searle et al. 2008). Data filters were applied requiring a minimum of two unique peptides per protein and probability thresholds to result in a $<0.1 \%$ peptide false discovery rate. Proteins that contained similar peptides and could not be differentiated on the basis of MS/MS analysis alone were grouped to satisfy the principles of parsimony.

Relative quantitation was determined using both spectral counting (SpC) and average MS/MS total ion current (Freund and Prenni 2013). Data were normalized using the default method in the Scaffold 3 software. A $t$-test was used to determine proteins that were significantly different in abundance 
Table 2: Distal femoral trabecular bone properties were not different between hibernating and summer marmots

\begin{tabular}{llccccc}
\hline & & \multicolumn{2}{c}{ Hibernation } & & \multicolumn{2}{c}{ Summer } \\
\cline { 3 - 4 } Trabecular bone property & \multicolumn{1}{c}{ Unit } & Mean & SE & & Mean & SE \\
\hline Bone volume fraction & $\%$ & 18.8 & 2.4 & 23.6 & 3.1 & .26 \\
No. & $1 / \mathrm{mm}$ & 2.70 & .17 & .81 & .19 & .67 \\
Thickness & $\mathrm{mm}$ & .10 & .003 & .11 & .006 & .11 \\
Separation & $\mathrm{mm}$ & .37 & .03 & .35 & .03 & .53 \\
Tissue mineral density & $\mathrm{mg} \mathrm{HA} / \mathrm{cm}^{3}$ & 715 & 11 & 712 & 7 & .83 \\
\hline
\end{tabular}

Note. HA, hydroxyapatite.

between seasons $(P<0.05)$. The resulting list of significantly different proteins was further filtered by the following criteria: proteins must be present in a minimum of two out of three biological replicates for a given group, and the total normalized spectral counts for a given group must be $>10$. Pseudo values were added ( +1 for spectral counting and $+1,000$ for average MS/MS total ion current) before fold change calculations to eliminate zero values.

\section{Statistical Analyses}

GraphPad Prism was used to conduct $t$-tests to compare the outcome variables. Grubb's test was used to detect significant outliers. A significance level of 0.05 was used for all statistical analyses.

\section{Results}

Microcomputed Tomography

Femur length was not different $(P=0.9)$ between hibernating $(74 \pm 3.5 \mathrm{~mm})$ and summer $(75 \pm 3.8 \mathrm{~mm})$ marmots. Body mass was not different $(P=0.3)$ between hibernating $(3.0 \pm 0.5 \mathrm{~kg})$ and summer $(3.3 \pm 0.1 \mathrm{~kg})$ marmots. Trabecular bone volume fraction, number, thickness, separation, and trabecular tissue mineral density were not different between seasons (table 2).

\section{Trabecular Histomorphometry}

No double labels were observed in the trabeculae of the distal femoral metaphysis in hibernating marmots (table 3; fig. 4A). In summer animals, double labels accounted for $6.75 \%$ of total trabecular BS, and they had significantly greater MAR $(P<.001)$ than hibernators. Despite the lack of dLS during hibernation, single labels were similar in proportion to total trabecular surface in both hibernating $(29.3 \%)$ and nonhibernating (32.7\%) marmots, indicating that trabecular bone mineral deposition was not absent during hibernation (fig. 4A). In the summer, there was significantly more mineralizing surface (table 3), including the presence of double labels (fig. $4 B$ ). One active animal showed a particularly high degree of mineralizing activity and had outlying values for MS/BS (52.8\%) and

Table 3: Bone remodeling activity decreased and marrow adiposity increased in distal femoral metaphyses of hibernating compared with summer marmots

\begin{tabular}{|c|c|c|c|c|c|c|}
\hline \multirow[b]{2}{*}{ Stain and variable } & \multirow[b]{2}{*}{ Unit } & \multicolumn{2}{|c|}{ Hibernation } & \multicolumn{2}{|c|}{ Summer } & \multirow[b]{2}{*}{$P$} \\
\hline & & Mean & SE & Mean & SE & \\
\hline \multicolumn{7}{|l|}{ Calcein: } \\
\hline sLS/BS & $\%$ & 29.4 & 3.3 & 32.7 & 3.1 & .47 \\
\hline $\mathrm{dLS} / \mathrm{BS}$ & $\%$ & .0 & .0 & 6.75 & .54 & $<.0001$ \\
\hline Mineralizing surface/BS & $\%$ & 14.8 & 1.6 & 22.8 & 2.4 & .03 \\
\hline MAR & $\mu / \mathrm{d}$ & .0 & .0 & 1.41 & .10 & $<.0001$ \\
\hline \multicolumn{7}{|l|}{ VonKossa/MacNeal's: } \\
\hline Osteoid width & $\mu \mathrm{m}$ & 1.05 & .23 & 1.57 & .11 & .08 \\
\hline Osteoid surface/BS & $\%$ & 3.2 & .8 & 10.6 & 1.5 & .002 \\
\hline Osteoblast no./BS & $1 / \mathrm{mm}$ & 1.00 & .18 & 3.65 & .41 & .0004 \\
\hline Area of adipocytes & $\%$ & 88.7 & 2.0 & 66.9 & 7.2 & .02 \\
\hline \multicolumn{7}{|l|}{ TRAP: } \\
\hline Eroded surface/BS & $\%$ & 2.19 & .55 & 4.49 & 2.35 & .37 \\
\hline Osteoclast surface/BS & $\%$ & 1.03 & .33 & 2.50 & 1.19 & .27 \\
\hline Osteoclast no./BS & $1 / \mathrm{mm}$ & .36 & .10 & .94 & .39 & .19 \\
\hline
\end{tabular}

Note. sLS, length of single-labeled surface; BS, bone surface; dLS, length of double-labeled surface; MAR, mineral apposition rate; TRAP, tartrate-resistant acid phosphatase. 



Figure 4. Calcein labels of the distal femoral metaphysis from hibernating $(A)$ and summer active $(B)$ marmots. Scale bars $=200 \mu \mathrm{m}$.

dLS/BS (35.1\%); therefore, it was excluded from the statistical analyses.

The relative osteoid surface and osteoblast numbers were significantly $(P<0.002)$ lower in hibernating animals than in active animals (table 3 ). The same active animal that showed exceptionally high mineralizing activity also had outlying values for osteoid surface $(33.8 \%)$ and osteoblast number $(10.1$ osteoblasts/mm) and therefore was not included in the statistical analyses. There were significantly more osteoblasts $(P=0.0004)$ and more osteoid surface $(P=0.002)$ in summer animals, but osteoid width was not different $(P=0.08)$ between seasons. There was a significantly $(P=0.019)$ higher percentage of the area of adipocytes occupying the trabecular bone marrow area in hibernators than in summer animals. There were no significant differences in the histomorphometric indices of osteoclasts and bone resorption activity between seasons (table 3).

\section{Cortical Histomorphometry}

As in trabecular bone, double calcein labels were not detectable on the periosteal surface of the midfemur cortical bone of hibernators. In summer animals, double labels were found on $22.7 \% \pm 11.8 \%$ of the periosteal surface (table 4 ). Thus, the periosteal MAR was significantly $(P=0.0004)$ greater in summer animals than in hibernators. A significantly $(P=$ 0.0103 ) greater percentage of the periosteal surface had single calcein labels in summer animals compared with hibernators. Double calcein labels were found on only two of the five hibernating marmots, and only a very small percentage $(0.34 \% \pm$ $0.32 \%)$ of the endosteal surface in those animals had double labels. Summer marmots had significantly $(P=0.024)$ more endosteal dLS than hibernators. One hibernating animal had an outlying value for endosteal sLS/BS (76.9\%); therefore, it

Table 4: Cortical bone mineralization was suppressed during hibernation

\begin{tabular}{|c|c|c|c|c|c|c|}
\hline \multirow[b]{2}{*}{ Surface and variable } & \multirow[b]{2}{*}{ Unit } & \multicolumn{2}{|c|}{ Hibernation } & \multicolumn{2}{|c|}{ Summer } & \multirow[b]{2}{*}{$P$} \\
\hline & & Mean & SE & Mean & SE & \\
\hline \multicolumn{7}{|l|}{ Periosteal: } \\
\hline sLS/BS & $\%$ & 3.1 & 1.7 & 31.7 & 8.4 & .01 \\
\hline $\mathrm{dLS} / \mathrm{BS}$ & $\%$ & .00 & .00 & 22.7 & 11.8 & .09 \\
\hline MAR & $\mu \mathrm{m} / \mathrm{d}$ & .00 & .00 & 1.26 & .21 & .0004 \\
\hline \multicolumn{7}{|l|}{ Endosteal: } \\
\hline sLS/BS & $\%$ & 23.2 & 9.8 & 18.4 & 6.9 & .69 \\
\hline $\mathrm{dLS} / \mathrm{BS}$ & $\%$ & .34 & .32 & 53.9 & 19.4 & .024 \\
\hline MAR & $\mu \mathrm{m} / \mathrm{d}$ & .89 & .004 & 1.33 & .18 & .21 \\
\hline
\end{tabular}

Note. sLS, length of single-labeled surface; BS, bone surface; dLS, length of double-labeled surface; MAR, mineral apposition rate. 
Table 5: Cortical bone osteocyte lacunar properties did not vary between seasons

\begin{tabular}{llllllll}
\hline & & \multicolumn{2}{c}{ Hibernation } & & \multicolumn{2}{c}{ Summer } & \\
\cline { 3 - 4 } Variable & Unit & Mean & SE & & Mean & SE & $P$ \\
\hline Area & $\mu \mathrm{m}^{2}$ & 22.2 & .9 & & 21.0 & 1.0 & .39 \\
Porosity & $\%$ & .21 & .02 & & .25 & .03 & .29 \\
Density & $\mathrm{mm}^{-2}$ & 99 & 11 & & 120 & 9 & .18 \\
\hline
\end{tabular}

was excluded from the statistical analyses. There was no seasonal difference $(P=0.69)$ in endosteal sLS/BS. Cortical bone osteocyte lacunar properties were not different between seasons (table 5).

\section{Femoral Cross-Sectional and Mechanical Properties}

There were no significant differences in the cross-sectional or mechanical properties between marmot femurs collected during hibernation or in the summer when marmots were physically active (table 6).

\section{Proteomics}

In total, 506 proteins were identified. Of these, 40 were significantly different in abundance between active and hibernating marmots (table 7). The most highly differentially abundant protein between seasons was monoaglycerol lipase (MGL), which was 98 -fold higher in hibernating marmots compared with summer marmots. Other highly differentially abundant proteins during hibernation included those involved in immune system function (e.g., immunoglobulin heavy chain $\mathrm{C}$ and $\mathrm{C} 1$ esterase inhibitor), iron and copper transport and reduction (e.g., transferrin receptor protein 1/CD71 antigen, serotransferrin, and amyloid beta A4), and bone remodeling and mineralization (e.g., collagenase 3, osteomodulin, and osteonectin).

\section{Discussion}

Bone disuse due to physical inactivity in traditional experimental lab animals and humans increases bone resorption and uncouples bone formation from resorption, leading to bone loss and increased fracture risk (Vestergaard et al. 1998; Wang et al. 2001; Li et al. 2005). Hibernating bears do not show bone loss during prolonged physical inactivity (McGee et al. 2007a, 2007b, 2008; McGee-Lawrence et al. 2009a, 2009b), and they decrease intracortical remodeling activity to about $25 \%$ of summer levels while maintaining balanced bone formation/ resorption (McGee et al. 2008). However, the biological mechanisms that regulate bone metabolism in hibernating mammals are not well understood. Marmots are also capable of preserving bone geometrical and mechanical properties, despite extended periods of mechanical disuse associated with hibernation (Wojda et al. 2012). Hibernating bears and rodents have very different physiological changes during hibernation. For example, hibernating bears reduce metabolism to $25 \%$ of summer basal metabolic levels (Toien et al. 2011), whereas hibernating rodents reduce it to $2 \%-5 \%$ of summer levels (Carey et al. 2003). We hypothesized that bone properties would be preserved during hibernation and that bone remodeling in hibernating marmots would be reduced to similar levels as overall metabolism (i.e., 5\%). There were no differences in cortical bone geometry and strength or trabecular bone architecture between hibernating and physically active marmots. Mineralization of the cortical bone on the periosteal surface of the midfemur was reduced to $\leq 10 \%$ of summer levels during hibernation, and endosteal double calcein labels were reduced to $0.6 \%$ of summer levels, but endosteal single calcein labels were not different between seasons. Trabecular single calcein labels were also not different between seasons, but double calcein labels were not found in hibernators. The trabecular bone mineralizing surface during hibernation was $65 \%$ of summer levels. Elucidating the mechanisms that regulate bone metabolism in hibernators may lead to a better understanding of how physiological processes have evolved to

Table 6: Midfemoral cortical bone cross-sectional and mechanical properties did not vary between seasons

\begin{tabular}{llllllll}
\hline & & \multicolumn{2}{c}{ Hibernation } & & \multicolumn{2}{c}{ Summer } & \\
Variable & Unit & Mean & SE & & Mean & SE & $P$ \\
\hline Cross-sectional area & $\mathrm{mm}^{2}$ & 18.1 & 1.7 & & 17.8 & 1.3 & .88 \\
$I_{\max }$ & $\mathrm{mm}^{4}$ & 78.8 & 14.9 & & 73.1 & 16.7 & .8 \\
Toughness & $\mathrm{mJ} / \mathrm{mm}^{3}$ & 12.4 & 1.5 & & 12.6 & 2.6 & .95 \\
Resilience & $\mathrm{mJ} / \mathrm{mm}^{3}$ & 1.8 & .2 & & 2.3 & .3 & .17 \\
Ultimate stress & $\mathrm{MPa}$ & 240 & 12 & & 259 & 13 & .31 \\
Yield stress & $\mathrm{MPa}$ & 158 & 14 & & 193 & 18 & .17 \\
Elastic modulus & $\mathrm{GPa}$ & 10.7 & .7 & & 12.6 & 1.1 & .16 \\
\hline
\end{tabular}


Table 7: Significantly differentially abundant proteins in hibernating marmots

\begin{tabular}{|c|c|c|c|c|}
\hline Accession no. & Gene & Protein name & $P$ & Fold change \\
\hline AGS_17204 & MGLL & Monoglyceride lipase & .005 & 98.45 \\
\hline AGS_7048 & TFRC & Transferrin receptor protein $1 / C D 71$ antigen & .0022 & 67.75 \\
\hline AGS_5294 & LOC100134331 & Immunoglobulin heavy chain $\mathrm{C}$ & .002 & 61.41 \\
\hline AGS_7045 & VIT & Vitrin & .00047 & 9.99 \\
\hline AGS_13755 & NID1 & Entactin & .05 & 6.81 \\
\hline AGS_11451 & HABP2 & Hyaluronan-binding protein 2 & .045 & 6.52 \\
\hline AGS_7461 & $\mathrm{TF}$ & Serotransferrin & .034 & 5.77 \\
\hline AGS_11436 & MMP13 & Collagenase 3 & .049 & 5.68 \\
\hline AGS_8597 & SERPING1 & $\mathrm{C} 1$ esterase inhibitor & .027 & 5.49 \\
\hline AGS_13347 & APP & Amyloid beta A4 & .019 & 5.30 \\
\hline AGS_9005 & OLFML1 & Olfactomedin-like protein 1 & .02 & 5.11 \\
\hline AGS_10777 & LAMA4 & Laminin subunit alpha-4 & .04 & 5.06 \\
\hline AGS_15388 & APCS & amyloid P component & .014 & 4.44 \\
\hline AGS_13593 & ECM2 & Extracellular matrix protein 2 & .05 & 4.38 \\
\hline AGS_963 & SERPINA3 & Alphat-1-antichymotrypsin & .046 & 4.11 \\
\hline AGS_7977 & TNC & Tenascin & .042 & 3.36 \\
\hline AGS_13592 & OMD & Osteomodulin & .0027 & 2.45 \\
\hline AGS_11739 & SPARC & Osteonectin & .026 & 2.26 \\
\hline AGS_12568 & F9 & Coagulation factor IX & .05 & 1.65 \\
\hline AGS_15885 & F9 & Coagulation factor IX & .015 & 1.52 \\
\hline AGS_16726 & IGLL1 & Immunoglobulin lambda-like polypeptide 1 & .034 & 1.46 \\
\hline AGS_10257 & $\mathrm{F} 2$ & Prothromib & .03 & 1.38 \\
\hline AGS_2029 & PROC & Vitamin K-dependent protein C & .013 & 1.35 \\
\hline AGS_13104 & BGN & Biglycan & .0052 & 1.31 \\
\hline AGS_9920 & ATP5A1 & ATP synthase subunit alpha & .022 & 1.26 \\
\hline AGS_19052 & ANXA2 & Annexin A2 & .0095 & 1.16 \\
\hline AGS_13469 & HSPA5 & Heat shock $70-$ Kda protein 5 & .0078 & .79 \\
\hline AGS_4596 & MSN & Moesin & .038 & .69 \\
\hline AGS_15424 & RPL14 & Ribosomal protein L14 & .0033 & .68 \\
\hline AGS_1339 & $\mathrm{P} 4 \mathrm{HB}$ & Protein disulfide-isomerase & .025 & .65 \\
\hline AGS_7088 & GAPDH & Glyceraldehyde-3-phosphase dehydrogenase & .035 & .60 \\
\hline AGS_15342 & HBA1 & Hemoglobin alpha & .049 & .54 \\
\hline AGS_15359 & PRDX1 & Peroxiredoxin 1 & .012 & .51 \\
\hline AGS_6496 & PDIA4 & Protein disulfide-isomerase A4 & .00011 & .40 \\
\hline AGS_18344 & TUBB2C & Tubullin beta & .013 & .31 \\
\hline AGS_4792(+1) & A1BG & Alpha 1-B glycoprotein & .026 & .25 \\
\hline AGS_15347 & GSTP1 & Glutahione S-transerase pi 1 & .045 & .24 \\
\hline AGS_15707 & TPI1 & Triosephosphate isomerase 1 & .0054 & .22 \\
\hline AGS_15341 & HSPA8 & Heat shock 70 -Kda protein 8 & .0029 & .13 \\
\hline AGS_10743 & VIM & Vimentin & .038 & .01 \\
\hline
\end{tabular}

${ }^{a}$ Hibernating/active.

conserve metabolic energy without compromising bone integrity during hibernation. Forty proteins were differentially expressed in marmot bone during hibernation and may provide some insight on the mechanisms that regulate bone metabolism during hibernation. For example, we found that immunoglobulin heavy chain (a large polypeptide subunit of an antibody) was higher in bone samples in hibernating compared with active marmots. This is consistent with previous studies showing higher levels of immunoglobulin heavy chain in serum from hibernating compared with active bears (Chow et al. 2013), which may possibly help explain bears' ability for wound healing during hibernation (Nishio et al. 2009; Iaizzo et al. 2012). Our finding is also consistent with increased numbers of B-lymphocytes in blood of hibernating 13-lined ground squirrels compared with active squirrels (Bouma et al. 2013).

We found significant reductions in bone formation and mineralization and also changes in the bone proteome, despite the small sample size in our study. It is possible that the lack of changes in bone structural and mechanical parameters were due to low sample size and power. However, a recent high-powered 


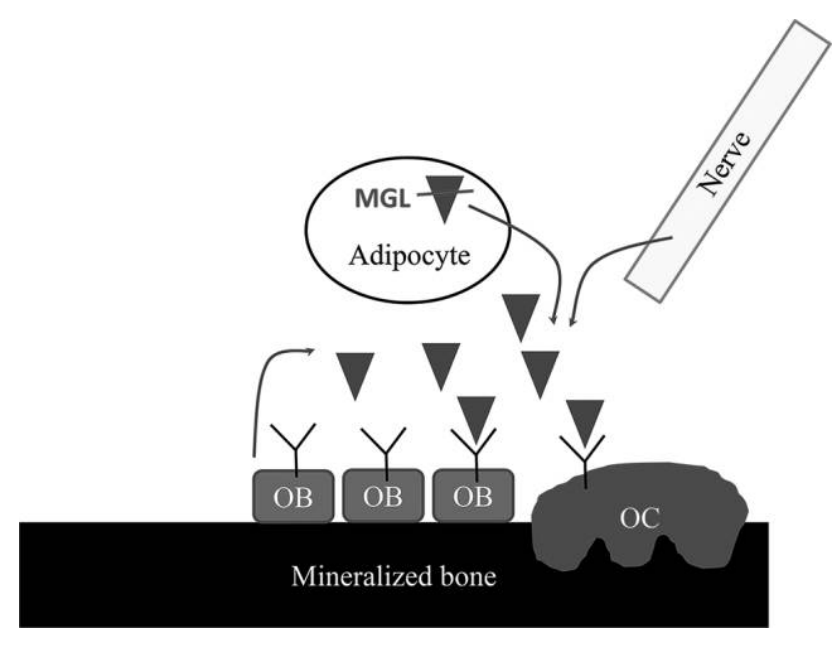

Figure 5. Endocannabinoid signaling in bone. Cannabinoid type 1 receptors on osteoblasts $(\mathrm{OB})$ and osteoclasts $(\mathrm{OC})$ bind the 2arachidonoyl glycerol (2-AG) ligand (triangles) from osteoblasts, nerve fibers, and adipocytes. Monoaglycerol lipase (MGL) may degrade 2-AG in adipocytes, bone cells, and nerve cells. A color version of this figure is available online.

study using bones from 66 wild marmots also found no adverse effects of hibernation on bone structural and mechanical parameters (Wojda et al. 2012). Our study had predominately female samples, so we were unable to determine whether there are sex-related differences in bone responses to hibernation. Double calcein labels were not found in trabecular bone or the periosteal surface of the cortical bone in midfemurs, and double calcein labels were found on only $0.34 \%$ of the endosteal surface of midfemurs. Thus, the MAR was calculated as 0 for trabecular and periosteal bone. However, since single calcein labels were detected on these surfaces, it is possible that the distance between the two calcein labels in hibernators was below the resolution of our imaging and that the actual MAR is not 0 but close to 0 . The marmots were synchronized in January so they would all be on the same torpor bout schedule, and necropsy could be scheduled for midtorpor as opposed to during interbout arousal, when metabolism increases for short durations. The stage of hibernation may contribute to variations in the physiology of bone remodeling processes (Doherty et al. 2014). It is possible that one or more of the marmots were out of sync and were entering or exiting a torpor bout (i.e., at a higher metabolic state) at the time of sample collection, and this may explain some of the outlying values we found.

Hibernation is an adaptation to extreme environmental conditions that limit food supplies, resulting in suppressed metabolism for the conservation of energy (Carey et al. 2003). Bone remodeling is an energy expensive process (Ishii et al. 2009). Thus, suppression of bone remodeling contributes to energy conservation in hibernating mammals. Bone remodeling evolved to serve numerous physiological functions, including organismal calcium homeostasis and mechanical homeostasis of bones (Dohertyetal.2015). Ourfindings on the architectural, geometrical, and mechanical properties of marmot bones indicate that bone remodeling processes maintain mechanical homeostasis during prolonged physical inactivity, unlike the disuse-induced bone loss that occurs in other animals (Gross and Rubin 1995; Houde et al. 1995; Li et al. 2005). The suppression of cortical bone remodeling seems to be consistent with global metabolic suppression in mammalian hibernators. Intracortical remodeling is suppressed to $25 \%$ of summer levels in hibernating bears (McGee et al. 2008),
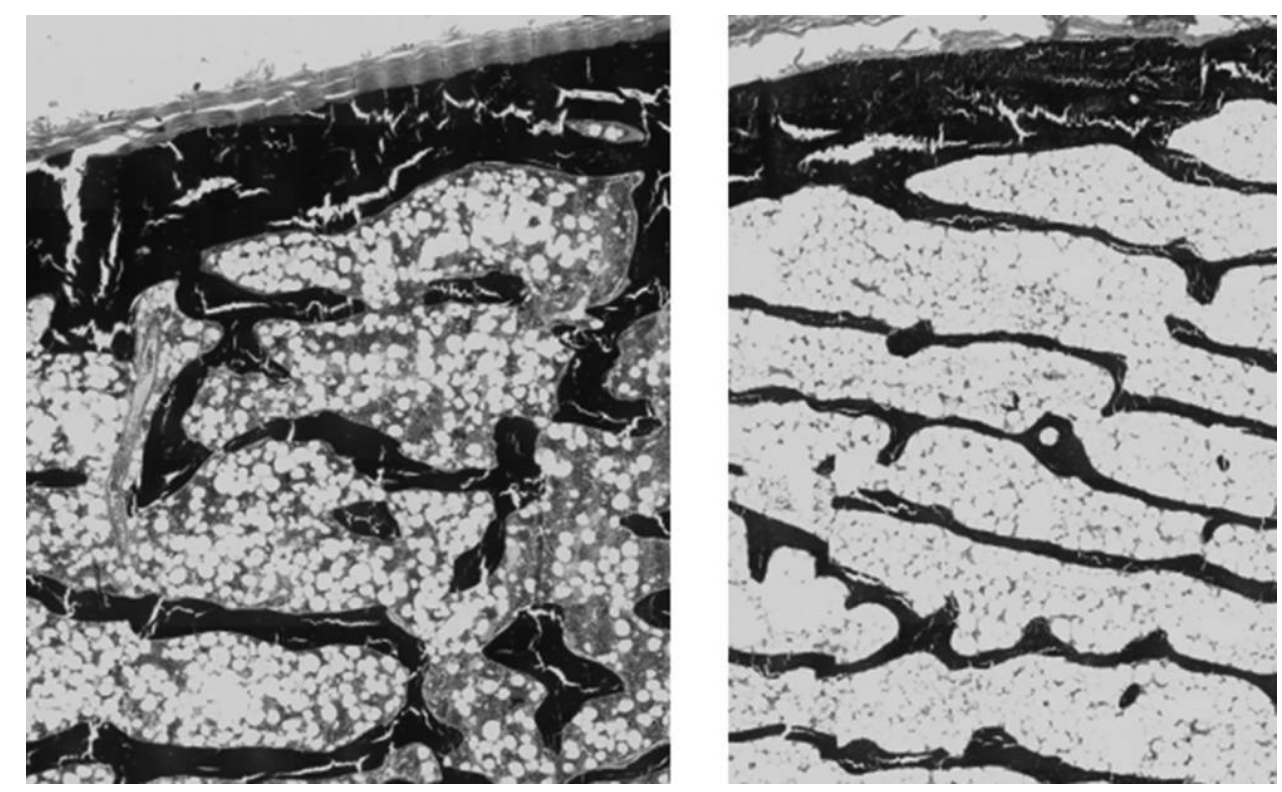

Figure 6. Bone marrow adiposity (white regions between black-stained bone tissue) is greater in hibernating marmots (right) than in summer marmots (left). A color version of this figure is available online. 
similar to the suppression of basal metabolic rate (Toien et al. 2011). Marmots are small enough in body mass that they only very rarely show intracortical remodeling (Wojda et al. 2012), and therefore intracortical remodeling was not evaluated in this study. Hibernating marmots showed a dramatic reduction in periosteal and endosteal mineralization (table 4). Periosteal single calcein labels in hibernators were reduced to $10 \%$ of summer levels, although endosteal single calcein labels were not different between seasons. Endosteal double calcein labels were reduced to $0.6 \%$ of summer levels, and double calcein labels were completely absent from the periosteal surface in hibernators. These findings are consistent with the reduction of hibernation metabolic rate to $5.5 \%$ of summer levels in marmots (Hock 1969). The suppression of trabecular bone remodeling, on the other hand, seems to be uncoupled from the reduction in basal metabolic rate in marmots. In the trabecular bone of hibernating marmots, mineralizing surface was $67 \%$ of summer levels, osteoid surface was $30 \%$, osteoblast number was $27 \%$, and osteoclast number was $38 \%$, although it did not reach statistical significance. It is possible that trabecular bone remodeling is not suppressed as much as basal metabolic rate in marmots because it is needed to play an important role in organismal calcium homeostasis. Osteoblasts metabolize fatty acids to provide energy for bone formation processes, and they may participate in the regulation of organismal energy homeostasis (Frey et al. 2015). The most highly differentially expressed protein between seasons was MGL (98-fold higher during hibernation). MGL is the enzyme that breaks down the endocannabinoid 2-arachidonoyl glycerol (2-AG; Labar et al. 2010). MGL may also be involved in regulating lipids used for energy metabolism by hydrolyzing monoglycerides in bone marrow adipocytes (Bolsoni-Lopes and Alonso-Vale 2015). 2-AG is a ligand for the cannabinoid type 1 receptor (CB1), which is found on bone cells (osteoblasts and osteoclasts), adipocytes, and nerve fibers that innervate bone (fig. 5). 2-AG is produced by osteoblasts in bone marrow (Idris and Ralston 2012) and in peripheral nerves (Tam et al. 2008). The trabecular bone samples in this study were sonicated to remove marrow and other soft tissues, so it is likely that the protein levels of MGL are due to production by osteoblasts, but it is possible there was some MGL from marrow adipocytes or nerve fibers as well. Activation of $\mathrm{CB} 1$ by 2-AG increases the number and activity of bone-forming osteoblasts and boneresorbing osteoclasts, whereas $\mathrm{CB} 1$ deficiency leads to decreased numbers of osteoblasts and osteoclasts and a striking increase in the number of bone marrow adipocytes (Idris and Ralston 2012). As in CB1 deficiency, hibernating marmots have a strikingly large accumulation of adipocytes in bone marrow (fig. 6; table 3) and low numbers of osteoblasts and osteoclasts compared with physically active marmots in the summer. Thus, the endocannabinoid system may be involved in regulating bone and energy metabolism in hibernating marmots by increasing adiposity and decreasing bone remodeling. This may be accomplished by reduced levels of $\mathrm{CB} 1$ expression and/or reduced levels of the 2-AG ligand via increased MGL (fig. 5).

In summary, we found that hibernating marmots - similar to hibernating black and grizzly bears-have evolved biological mechanisms to prevent bone loss during prolonged periods of physical inactivity and obesity. The suppression of bone remodeling during hibernation contributes to the conservation of metabolic energy during hibernation when food is unavailable. The mechanisms regulating bone metabolism in hibernators is not known, but these proteomics data raise the possibility that the endocannabinoid system is involved. Endocannabinoids are signaling molecules derived from fatty acids that appeared early in evolution and play important roles in regulating numerous physiological processes, including those that are altered in hibernation, such as bone, fat, and energy metabolism. Many tissues and organs that would normally be adversely affected by obesity and physical inactivity have integrative physiological relationships. For example, bone plays important physiological roles in reproduction and fat and energy metabolism, and these systems are all influenced by the endocannabinoid system. Thus, further investigation on the endocannabinoid system in hibernators may increase our understanding of the regulation of suppressed metabolism in many physiological systems during hibernation.

\section{Acknowledgments}

We thank Jacklyn Watson, Katherine Metoyer, and Kristyn Hosmer for contributions to data collection and animal care and maintenance. We also thank Margie Owen, Claire Tucker, Ryan Curtis, Melanie Hoobler, and Ashley Heim for their participation in animal trapping and/or sample collection at necropsy.

\section{Literature Cited}

Armitage K.B., D.T. Blumstein, and B.C. Woods. 2003. Energetics of hibernating yellow-bellied marmots (Marmota flaviventris). Comp Biochem Physiol A Mol Integr Physiol 134:101-114.

Baek K. and S.A. Bloomfield. 2009. Beta-adrenergic blockade and leptin replacement effectively mitigate disuse bone loss. L Bone Miner Res 24:792-799.

Bolsoni-Lopes A. and M.I. Alonso-Vale. 2015. Lipolysis and lipases in white adipose tissue: an update. Arch Endocrinol Metab 59:335-342.

Bouma H.R., R.H. Henning, F.G. Kroese, and H.V. Carey. 2013. Hibernation is associated with depression of T-cell independent humoral immune responses in the 13-lined ground squirrel. Dev Comp Immunol 39:154-160.

Carey H.V., M.T. Andrews, and S.L. Martin. 2003. Mammalian hibernation: cellular and molecular responses to depressed metabolism and low temperature. Physiol Rev 83:1153-1181.

Chow B.A., S.W. Donahue, M.R. Vaughan, B. McConkey, and M.M. Vijayan. 2013. Serum immune-related proteins are differentially expressed during hibernation in the American black bear. PLoS ONE 8:e66119.

Dempster D.W., J.E. Compston, M.K. Drezner, F.H. Glorieux, J.A. Kanis, H. Malluche, P.J. Meunier, S.M. Ott, R.R. Recker, and A.M. Parfitt. 2013. Standardized nomenclature, symbols, and units for bone histomorphometry: a 2012 update of the 
report of the ASBMR Histomorphometry Nomenclature Committee. LBone Miner Res 28:2-17.

Doherty A.H., G.L. Florant, and S.W. Donahue. 2014. Endocrine regulation of bone and energy metabolism in hibernating mammals. Integr Comp Biol 54:463-483.

Doherty A.H., J.D. Frampton, and C.J. Vinyard. 2012. Hibernation does not reduce cortical bone density, area or second moments of inertia in woodchucks (Marmota monax). I Morphol 273:604-617.

Doherty A.H., C.K. Ghalambor, and S.W. Donahue. 2015. Evolutionary physiology of bone: bone metabolism in changing environments. Physiology 30:17-29.

Floyd T., R.A. Nelson, and G.F. Wynne. 1990. Calcium and bone metabolic homeostasis in active and denning black bears (Ursus americanus). Clin Orthop Rel Res 255:301-309.

Freund D.M. and J.E. Prenni. 2013. Improved detection of quantitative differences using a combination of spectral counting and MS/MS total ion current. LProteome Res 12:1996-2004.

Frey J.L., Z. Li, J.M. Ellis, Q. Zhang, C.R. Farber, S. Aja, M.J. Wolfgang, T.L. Clemens, and R.C. Riddle. 2015. Wnt-Lrp5 signaling regulates fatty acid metabolism in the osteoblast. Mol Cell Biol 35:1979-1991.

Gross T.S. and C.T. Rubin. 1995. Uniformity of resorptive bone loss induced by disuse. I Orthop Res 13:708-714.

Hock R.J. 1969. Thermoregulatory variations of high-altitude hibernators in relation to ambient temperature, season, and hibernation. Fed Proc 28:1047-1052.

Houde J.P., L.A. Schulz, W.J. Morgan, T. Breen, L. Warhold, G.K. Crane, and D.T. Baran. 1995. Bone mineral density changes in the forearm after immobilization. Clin Orthop Relat Res 317:199-205.

Iaizzo P.A., T.G. Laske, H.J. Harlow, C.B. McClay, and D.L. Garshelis. 2012. Wound healing during hibernation by black bears (Ursus americanus) in the wild: elicitation of reduced scar formation. Integr Zool 7:48-60.

Idris A.I. and S.H. Ralston. 2012. Role of cannabinoids in the regulation of bone remodeling. Front Endocrinol (Lausanne) 3:136.

Ishii K.A., T. Fumoto, K. Iwai, S. Takeshita, M. Ito, N. Shimohata, H. Aburatani, et al. 2009. Coordination of PGC-1beta and iron uptake in mitochondrial biogenesis and osteoclast activation. Nat Med 15:259-266.

Jiang X., M. Ye, X. Jiang, G. Liu, S. Feng, L. Cui, and H. Zou. 2007. Method development of efficient protein extraction in bone tissue for proteome analysis. IProteome Res 6:2287-2294.

Kall L., J.D. Storey, M.J. MacCoss, and W.S. Noble. 2008. Assigning significance to peptides identified by tandem mass spectrometry using decoy databases. LProteome Res 7:29-34.

Kaneps A.J., S.M. Stover, and N.E. Lane. 1997. Changes in canine cortical and cancellous bone mechanical properties following immobilization and remobilization with exercise. Bone 21:419-423.

Keller A., A.I. Nesvizhskii, E. Kolker, and R. Aebersold. 2002. Empirical statistical model to estimate the accuracy of peptide identifications made by MS/MS and database search. Anal Chem 74:5383-5392.
Labar G., J. Wouters, and D.M. Lambert. 2010. A review on the monoacylglycerol lipase: at the interface between fat and endocannabinoid signalling. Curr Med Chem 17:2588-2607.

Li C.Y., C. Price, K. Delisser, P. Nasser, D. Laudier, M. Clement, K.J. Jepsen, and M.B. Schaffler. 2005. Long-term disuse osteoporosis seems less sensitive to bisphosphonate treatment than other osteoporosis. LBone Miner Res 20:117-124.

Li X.J., W.S. Jee, S.Y. Chow, and D.M. Woodbury. 1990. Adaptation of cancellous bone to aging and immobilization in the rat: a single photon absorptiometry and histomorphometry study. Anat Rec 227:12-24.

McGee M.E., K.W. Magic, D.L. Miller, A.J. Maki, and S.W. Donahue. 2007a. Black bear femoral porosity decreases and mechanical properties increase with age despite annual periods of disuse (hibernation). Eng Fract Mech 74:1942-1952.

McGee M.E., A.J. Maki, S.E. Johnson, O.L. Nelson, C.T. Robbins, and S.W. Donahue. 2008. Decreased bone turnover with balanced resorption and formation prevent cortical bone loss during disuse (hibernation) in grizzly bears (Ursus arctos horribilis). Bone 42:396-404.

McGee M.E., D.L. Miller, J. Auger, H.L. Black, and S.W. Donahue. 2007b. Black bear femoral geometry and cortical porosity are not adversely affected by ageing despite annual periods of disuse (hibernation). LAnat 210:160-169.

McGee-Lawrence M., P. Buckendahl, C. Carpenter, K. Henriksen, M. Vaughan, and S. Donahue. 2015. Suppressed bone remodeling in black bears conserves energy and bone mass during hibernation. LExp Biol 218:2067-2074.

McGee-Lawrence M.E., H.V. Carey, and S.W. Donahue. 2008. Mammalian hibernation as a model of disuse osteoporosis: the effects of physical inactivity on bone metabolism, structure, and strength. Am J Physiol 295:R1999-R2014.

McGee-Lawrence M.E., D.M. Stoll, E.R. Mantila, B.K. Fahrner, H.V. Carey, and S.W. Donahue. 2011. Thirteen-lined ground squirrels (Ictidomys tridecemlineatus) show microstructural bone loss during hibernation but preserve bone macrostructural geometry and strength. L Exp Biol 214:1240-1247.

McGee-Lawrence M.E., S.J. Wojda, L.N. Barlow, T.D. Drummer, K. Bunnell, J. Auger, H.L. Black, and S.W. Donahue. 2009a. Six months of disuse during hibernation does not increase intracortical porosity or decrease cortical bone geometry, strength, or mineralization in black bear (Ursus americanus) femurs. LBiomech 42:1378-1383.

McGee-Lawrence M.E., S.J. Wojda, L.N. Barlow, T.D. Drummer, A.B. Castillo, O. Kennedy, K.W. Condon, et al. $2009 b$. Grizzly bears (Ursus arctos horribilis) and black bears (Ursus americanus) prevent trabecular bone loss during disuse (hibernation). Bone 45:1186-1191.

Nelson R.A., T.D. Beck, and D.L. Steiger. 1984. Ratio of serum urea to serum creatinine in wild black bears. Science 226:841-842.

Nishio N., S. Ito, H. Suzuki, and K. Isobe. 2009. Antibodies to wounded tissue enhance cutaneous wound healing. Immunology 128:369-380.

Rantakokko J., H. Uusitalo, T. Jamsa, J. Tuukkanen, H.T. Aro, and E. Vuorio. 1999. Expression profiles of mRNAs for osteoblast and osteoclast proteins as indicators of bone loss in 
mouse immobilization osteopenia model. [Bone Miner Res 14: 1934-1942.

Rubin C., T. Gross, Y.X. Qin, S. Fritton, F. Guilak, and K. McLeod. 1996. Differentiation of the bone-tissue remodeling response to axial and torsional loading in the turkey ulna. L Bone Joint Surg Am 78:1523-1533.

Rubin C.T., G.W. Pratt Jr., A.L. Porter, L.E. Lanyon, and R. Poss. 1988. Ultrasonic measurement of immobilization-induced osteopenia: an experimental study in sheep. 42:309-312.

Rudnick P.A., K.R. Clauser, L.E. Kilpatrick, D.V. Tchekhovskoi, P. Neta, N. Blonder, D.D. Billheimer, et al. 2010. Performance metrics for liquid chromatography-tandem mass spectrometry systems in proteomics analyses. Mol Cell Proteomics 9:225241.

Searle B.C., M. Turner, and A.I. Nesvizhskii. 2008. Improving sensitivity by probabilistically combining results from multiple MS/MS search methodologies. LProteome Res 7:245-253.

Seger R.L., R.A. Cross, C.J. Rosen, R.C. Causey, C.M. Gundberg, T.O. Carpenter, T.C. Chen, et al. 2011. Investigating the mechanism for maintaining eucalcemia despite immobility and anuria in the hibernating American black bear (Ursus americanus). Bone 49:1205-1212.

Shao C., Y. Liu, H. Ruan, Y. Li, H. Wang, F. Kohl, A.V. Goropashnaya, et al. 2010. Shotgun proteomics analysis of hibernating arctic ground squirrels. Mol Cell Proteomics 9:313-326.
Tam J., V. Trembovler, V. Di Marzo, S. Petrosino, G. Leo, A. Alexandrovich, E. Regev, et al. 2008. The cannabinoid CB1 receptor regulates bone formation by modulating adrenergic signaling. Faseb I 22:285-294.

Toien O., J. Blake, D.M. Edgar, D.A. Grahn, H.C. Heller, and B.M. Barnes. 2011. Hibernation in black bears: independence of metabolic suppression from body temperature. $\underline{\text { Sci- }}$ ence 331:906-909.

Utz J.C., S. Nelson, B.J. O’Toole, and F. van Breukelen. 2009. Bone strength is maintained after 8 months of inactivity in hibernating golden-mantled ground squirrels, Spermophilus lateralis. I Exp Biol 212:2746-2752.

Vestergaard P., K. Krogh, L. Rejnmark, and L. Mosekilde. 1998. Fracture rates and risk factors for fractures in patients with spinal cord injury. Spinal Cord 36:790-796.

Wang C.M., Y. Chen, M.J. DeVivo, and C.T. Huang. 2001. Epidemiology of extraspinal fractures associated with acute spinal cord injury. Spinal Cord 39:589-594.

Wojda S.J., M.E. McGee-Lawrence, R.A. Gridley, J. Auger, H.L. Black, and S.W. Donahue. 2012. Yellow-bellied marmots (Marmota flaviventris) preserve bone strength and microstructure during hibernation. Bone 50:182-188.

Young D.R., W.J. Niklowitz, and C.R. Steele. 1983. Tibial changes in experimental disuse osteoporosis in the monkey. Calcif Tissue Int 35:304-308. 